\title{
Introducing Health-Climate-Economics and Rapid Viability Test for Candidate Solutions as a Tool for Automated Healthcare Procurement and Evaluation
}

\author{
Alali, E. \\ Axelisys Limited, 2020 \\ (orcid https://orcid.org/0000-0001-6431-6176) \\ ethar@axelisys.co.uk
}

\begin{abstract}
The impact of the climate on the health of populations is now reaching the mainstream. The impact of health system decisions on the lives of its patients has been studied through several analyses and research projects that identified direct, unequivocal links between healthcare services and the effect on healthcare demand of those same services.

The UK NHS contributes the equivalent of 24 million tonnes of $\mathrm{CO} 2$ greenhouse gases to the climate footprint of the UK. This is $5.4 \%$ of the entire UK's Carbon footprint and is a value bigger than 2.8 million UK households or the entire emissions of the countries of Jordan, Croatia and Estonia. It is the UK's biggest single emitter of $\mathrm{CO} 2$ and consumer of single use plastics. Directly contributing to the deaths of 40,000 people through respiratory disorders, 900 through heatstroke at a cost of almost $£ 400$ million annually.

The majority of the NHS carbon footprint (19 million tonnes of CO2) come through the NHS supply chain with only 5.5 million tonnes resulting from internal activity. Suggesting the biggest impact will be driven through changes to procurement.

Procurement currently assigns a range of importance to health and economic factors. Most procurement is conducted with an asymmetric split of quality and cost. 80:20, 70:30 or 60:40 ratios of quality and cost, while the NHS evaluates the evidence base by using Quality Adjusted Life Years and Disability Adjusted Life Years, both of which evaluate the gain the technology provides, but does not include the impacts downstream of the patient, which result in this emissions footprint that in turn appears as A\&E and healthcare demand.

Together, these factors suggest current deployed health-economic models are not sufficient to catch the failure demand nor assess the lifecycle impact of climate effects on the service itself, via the population. This paper explores an alternative model that accounts for the impact of climate on the health-economic models and constructs an evaluation framework for supplier assessment.
\end{abstract}

Keywords: health-economics, sustainability, climate change, healthcare, circular economics, procurement, healthcare procurement, health-climate-economic modelling, sustainability in health procurement 


\section{Background}

Health-economic (1) models have become a mainstay of healthcare business cases for medicines and medical devices. Providing health evaluators with the proof and confidence of the product and form part of the latest best practise for bids to the NHS Category Towers (2).

The NHS published its decarbonisation plan in October 2020 acknowledging the scale of the problem and the link between climate and health impacts. Solidifying the work of Paavola (3) identifying and assessing the impact of climate change on the health of the UK population. Inexorably linking climate change with immediate effects from air quality, skin cancer and heat impacts that exacerbating existing health inequalities.

Yet, health-economics only covers the front-loaded cost of resources and considers this against existing "do nothing" and competitor offerings, by contrasting total health benefit for every monetary unit spend, and its aggregation in contextually important timespan. Most notably disability $\left(\mathrm{DALY}, I_{D}\right)$ and quality adjusted life years (QALY, $I_{Q}$ ).

$$
\begin{aligned}
& \frac{d I_{D}}{d C}=\frac{d}{d C} I_{D}(C, t) \\
& \frac{d I_{Q}}{d C}=\frac{d}{d C} I_{Q}(C, t) \\
& \text { Where } \\
& C=\text { cost factor } \\
& I=\text { health Impact } \\
& t
\end{aligned}
$$

Health economic models cover the front-loaded purchase of equipment, medicines and devices. However, once the health outcome has been achieved, there is no accounting for the impact that delivery transit and disposal of that equipment has on the future health of individuals. Despite the demonstrable climate link, which may have an impact on the patient in future, or other patients. As identified in Paavola.

This creates a disconnect between the perceived impact of the model, its use within healthcare settings and the actual impact purchases have on patients health through readmissions and retreatments.

Connecting the effect of climate impacts $(\mathrm{P})$, we can derive the impact of that procurement, which itself is with respect to cost. Implying the infinitesimals can be rewritten using the chain rule as:

$$
\begin{aligned}
& \frac{d I_{k}}{d C}=\frac{d I_{k}}{d P} \frac{d P}{d C}, k \in\{Q, D\} \\
& D=D A L Y \\
& Q=Q A L Y \\
& P=\text { Climate impact }
\end{aligned}
$$

This provides a convenient representation of the relationship between the health impact (in QALY and DALY) and both the climate impact on health and the cost of that climate impact.

Where the production of medical goods is remote, several components cease to become negligible in their contribution to the other dependent variables. Impacting in both climate and financial terms.

1. Raw materials (R)

2. Manufacturing (F)

3. Freight \& Logistics (L)

4. Disposal (D)

Simplifying to:

$$
P=\sum_{i \in\{R, F, L, D\}} P_{S}
$$

\section{Where}

$R=$ Raw material

$F=$ Manufacturing

$L=$ Freight\& logistics

$D=$ Disposal

Each of the processes can be further decomposed into their constituent parts.

\section{Raw Material}

Each extraction and process in raw materials production for care equipment has a footprint of its own, that's often missed in lifecycle and impact assessments that only measure from the manufacturer, not the raw material supplier. Multi-material equipment has the added complication of multiple processing stages and transit between sites (r).

$$
P_{R}=\sum_{r=1}^{n}\left(P_{R, r}+L_{R, r}\right)
$$

\section{Illustration}

$4 \mathrm{~kg}$ of oil creates an average of $1 \mathrm{~kg}$ of plastics. The annual impact of the extraction, containment, freight, cracking and shipping is 1.27 Gigatonnes of $\mathrm{CO} 2$ equivalent that becomes NHS equipment annually. Resultantly, the identified climate impact of the NHS itself, is an underestimate, when considered from the source.

Contrast this with bioplastics, where $1.6 \mathrm{~kg}$ of crop waste/biomass creates $1 \mathrm{~kg}$ of plastics, which also use less than half the energy to process. Allowing factories to run on much less power.

\section{Manufacturing}

Factory energy consumption and emissions are not insignificant. Most PPE factories consume 10 megawatt hours of energy each year (4). Textile consumption is the third highest Carbon producer behind oil and gas industries. 
This has significant ramifications for the choice of reusable mask but creates a very large carbon footprint for the creation of typical PPE. FFP3 masks

\begin{tabular}{|l|c|c|c|}
\hline Item & $\begin{array}{c}\text { Weight } \\
(\mathrm{g})\end{array}$ & $\begin{array}{c}\text { Energy } \\
\text { Consumption } \\
(\mathrm{kWh})\end{array}$ & $\begin{array}{c}\text { Emissions } \\
\text { including } \\
\text { transit } \\
(\mathrm{gCO})\end{array}$ \\
\hline Face visor & 81 & 0.6667 & 405 \\
\hline Mask & 12 & $0.0342 \mathrm{kWh}$ & 50 \\
\hline Gloves & 7 & $0.0003 \mathrm{kWh}$ & 30 \\
\hline
\end{tabular}

Given a full consignment is a maximal best-case scenario that optimises shipping. The resulting impact, including transit, provides us with the inequality:

$$
P_{F}>\sum_{S=1}^{k}\left(P_{F, S}+L_{F, S}\right)
$$

\section{Freight \& Logistics}

On top of the transit to ports, the separate step of exporting the goods carries an impact of its own. The degree of impact depends on the route and mode of transit. While air freight has largest footprint by far, the relatively low capacity and high cost make air transit infeasible for all but the smallest of loads. Most freight is carried by container ships and rail.

The climate impact of the freight of goods is regularly measured by weight in the literature (5). Necessitating a transformation between item and mass. Fortunately, this is a simple formulation of the number of items in a certain consignment mass (m) for each logistical impact:

$$
P_{L}=\frac{1}{m} \sum_{l=1}^{g} L_{l}
$$

The logistical impact itself, has a coefficient of emissions $(\alpha)$ measured in $\mathrm{kgCO} 2 / \mathrm{kg} / \mathrm{km}$ and ships a distance $(\mathrm{d})$ in each leg (l). Thus:

$$
\begin{gathered}
L_{l}=\alpha d_{l} \\
P_{L}=\frac{\alpha}{m} \sum_{l=1}^{g} d_{l}=\frac{\alpha}{m} d_{D}
\end{gathered}
$$

Where

$a=$ material emissions coefficient

$d=$ distance travelled

$m=$ mass of the material

\section{Disposal}

Disposal is composed of a transit impact and a final disposal impact. The latter may be landfill (f), incineration (b), or recycling (v). The impact of each type of final disposal has an emissions coefficient $\beta$ in $\mathrm{kg}$ of $\mathrm{CO} 2$ per $\mathrm{kg}$ of disposal.

$$
P_{D}=\sum_{u \in\{f, b, v\}}\left(\beta_{u} m_{u}+L_{D, u}\right)
$$

These create an aggregate equation of:

$$
\begin{gathered}
P>\sum_{r=1}^{n}\left(P_{R, r}+L_{R, r}\right)+\sum_{s=1}^{k}\left(P_{F, s}+L_{F, s}\right)+\frac{1}{m} \sum_{l=1}^{g} \alpha_{l} d_{l} \\
+\sum_{u \in\{f, b, v\}} \beta_{u} m_{u}+L_{D l}
\end{gathered}
$$

Which can be further simplified by collecting freight and logistics terms and adjusting by total travel distance.

$$
\begin{aligned}
P>\sum_{r=1}^{n} P_{R, r}+\alpha & \sum_{s=1}^{k} m_{s} d_{s}\left(P_{F, s}+\frac{1}{d_{D}}+\gamma\right)+\frac{\alpha}{m} d_{D} \\
& +\sum_{u \in\{f, b, v\}} \beta_{u} m_{u}+\alpha \sum_{r=1}^{n} m_{r} d_{r}
\end{aligned}
$$

Where logistics terms in each stage use similar freight options to long-haul freight, this can be simplified to a linear function of total distance $\left(d_{D}\right)$ and component product weight. Providing the maximal beneficial climate-cost at:

$$
\begin{gathered}
\frac{d P}{d C}=\frac{d}{d C}\left(\sum_{r=1}^{n} P_{R, r}+\alpha \sum_{s=1}^{k} m_{s} d_{s}\left(P_{F, s}+\frac{1}{d_{D}}+\gamma\right)+\frac{\alpha}{m} d_{D}\right. \\
\left.+\sum_{u \in\{f, b, v\}} \beta_{u} m_{u}+\alpha \sum_{r=1}^{n} m_{r} d_{r}\right)
\end{gathered}
$$

Where the minimal climate impact per unit-spend is achieved when $\frac{d P}{d C}=0$ and $\frac{d^{2} P}{d C^{2}}>0$.

\section{Health-Climate Impact}

The impact of climate change on UK residents and NHS demand by proxy, are well known and acknowledged (6). Emissions impact the quality of life of residents and also shorten average European lifespans by as much as 2.2 years with 1.7 of which being preventable (7). $33 \%$ of all new childhood asthma cases have been attributed to air pollution (8).

Health impacts come in a number of forms:

- Direct particulate or pollution impacts on respiratory conditions like asthma, bronchitis and COPD

- Heatstroke impacts on elderly UK residents

- Water-born disease via extreme events e.g. flooding 
Air pollution alone is the amalgamation of a number of factors:

- Raw material extraction

- Energy consumption

- Manufacturing emissions \& incineration including particulate matter

- Freight and Logistics emissions

- Public and private passenger transit

$$
\frac{d I_{k}}{d P}=X+E
$$

This material is directly proportional to the distance travelled. As tyres eject particulate matter that is 1,000 times worse than exhaust emissions (9).

Furthermore, the disposal impacts are limited to the energy use of recycling materials, not incineration nor landfill or shipping.

\section{Circularity}

The position presented in this paper would not be complete without an understanding of the effect of circular economics on the product lifecycle and health-climate-economics.

With health supply chains, there are several key areas circularity changes:

1. Minimises or eradicates extraction and processing of raw materials. Thereby reducing the emissions and stage 1 transit to 0 .

$$
\lim _{P_{r} \rightarrow 0} \sum_{r=1}^{n} P_{R, r}
$$

2. Removes or minimises the final disposal impact

$$
\left\{\forall u \mid u \in\{f, b, v\} \cdot \lim _{P_{D} \rightarrow 0} \sum \beta_{u} m_{u}+L_{D l}\right\}
$$

3. Reuses equipment that is capable of reuse requiring no logistics

$$
\left\{\forall u \mid u \in\{f, b, v\} \cdot \lim _{L_{D} \rightarrow 0} \sum \beta_{u} P_{D, r}+L_{D l}\right\}
$$

4. Increases the sanitisation impact $\left(\varepsilon_{l}\right)$ from reuse. Measured per item

$$
S=\sum_{l=1}^{g} \varepsilon_{l}
$$

This simplifies the $\mathrm{N}$-generation impact of reuse materials to:

$$
\begin{gathered}
N\left[\sum_{l=1}^{g}\left(\beta_{u} m_{u}+\varepsilon_{l}\right)\right] \\
\text { Where } \\
\quad N=\text { Total viable generations } \\
\varepsilon=\text { Sanitisation cost }
\end{gathered}
$$

A general case which includes the recycling and sterilisation of novel materials for reusable equipment with a lower climate impact than traditional materials, also reduces the impact of the initial raw material consumption by a factor $k$ in extraction and processing

$$
\begin{aligned}
\sum_{r=1}^{n}\left(\frac{1}{k} P_{R, r}+L_{R, r}\right) & +\sum_{s=1}^{k}\left(P_{F, s}+L_{F, S}\right) \\
& +N\left[\sum_{l=1}^{g}\left(\beta_{u} m_{u}+\varepsilon_{l}\right)\right]
\end{aligned}
$$

Compared to simply using recycled materials in the existing processing facilities, circular economics reduces emissions and disposal. That provides even greater savings over recycled equipment and contributes to cost savings through disposal and procurement savings, while materially improving the health-economic position above the recycled material footprint.

Also eradicating the need for new raw materials. In an idealised system with on-site recycling, this results in a climate impact of:

$$
\begin{array}{r}
P^{*}=\sum_{r=1}^{n} P_{R, r}+\alpha \sum_{s=1}^{k} m_{s} d_{s}\left(P_{F, s}+\gamma\right) \\
+N\left[\sum_{l=1}^{g}\left(\beta_{u} m_{u}+\varepsilon_{l}\right)\right]
\end{array}
$$

Where $h$ is the recycling energy consumption during processing.

This in turn, aggregating by processing and logistics terms, this creates a first-generation health-climate saving of:

$$
\begin{gathered}
P-P^{*}>\sum_{r=1}^{n}\left(\frac{k-1}{k} P_{R, r}\right)+\sum_{s=1}^{k}\left(P_{F, s}+L_{F, s}\right)+ \\
\frac{\alpha}{d_{D}} \sum_{s=1}^{k} m_{s} d_{s}+\frac{\alpha}{m} d_{D}+\sum_{u \in\{f, b, v\}} \beta_{u} P_{D, r}+\alpha \sum_{r=1}^{n} m_{r} d_{r} \\
-\sum_{l=1}^{g}\left(\beta_{g} m_{g}+\varepsilon_{l}\right)
\end{gathered}
$$

And N-th generation saving of:

$$
\begin{gathered}
N\left(P-P^{*}\right)>\sum_{r=1}^{n}\left(\frac{k-1}{k} P_{R, r}\right)+\sum_{s=1}^{k}\left(P_{F, S}+L_{F, S}\right)+ \\
N\left[\frac{\alpha}{d_{D}} \sum_{s=1}^{k} m_{s} d_{s}+\frac{\alpha}{m} d_{D}+\sum_{u \in\{f, b, v\}} \beta_{u} P_{D, r}+\alpha \sum_{r=1}^{n} m_{r} d_{r}\right. \\
\left.-\sum_{l=1}^{g}\left(\beta_{g} m_{g}+\varepsilon_{l}\right)\right]
\end{gathered}
$$




\section{Important Note: Plastics}

It is often stated that the price of "virgin plastics" is cheaper than recycling it. This is not the case systemically. It only applies when considering the plastic raw material manufacturing portion. Systemic consideration of delivery, logistics and energy use, which adds at least $10 \%$ to the price, without health impacts, is more expensive when evaluated robustly using the lifecycle system.

Furthermore, the cost of the original plastics, can be assessed against the lifecycle saving to maintain sufficient saving of both cost and climate impact.

\section{Combined Effects: Matrix Treatment}

Because of the interplay between the climate-health and health-cost metrics, the optimal health-climate-economic position maximises the QALY, with minimal climate impact and minimal cost, give the reduced wider health impact on the patient cohort due to reduced ecological pollutants.

In the 4-dimensional space, this manifests as a saddle point, which can be represented by the Hessian matrix and the mapping of partial derivatives with respect to climate (p), cost (c) and health (h):

$$
\begin{aligned}
& I \rightarrow f(c, p, h) \\
& \frac{\partial}{\partial x \partial y \partial z} f(x, y, z) \rightarrow f_{x y z} \\
& H(c, p, h)=\left(\begin{array}{lll}
f_{c c c} & f_{c p c} & f_{c p h} \\
f_{p c c} & f_{p p p} & f_{p c h} \\
f_{h c c} & f_{h p c} & f_{h h h}
\end{array}\right) \\
& h=\text { Health } Q A L Y \text { or } D A L Y \\
& c=\text { Financial cost } \\
& p=\text { Climate impact }
\end{aligned}
$$

Providing evaluators with a means to identify the necessary saddle point where Eigenvalues, $\lambda_{i}$ include both positive and negative Eigenvalues to attain the necessary saddle point of the idealised solution, for comparison to the "do nothing" acceptable solution sphere ( $\mathrm{C}$ in the figure 1$)$.

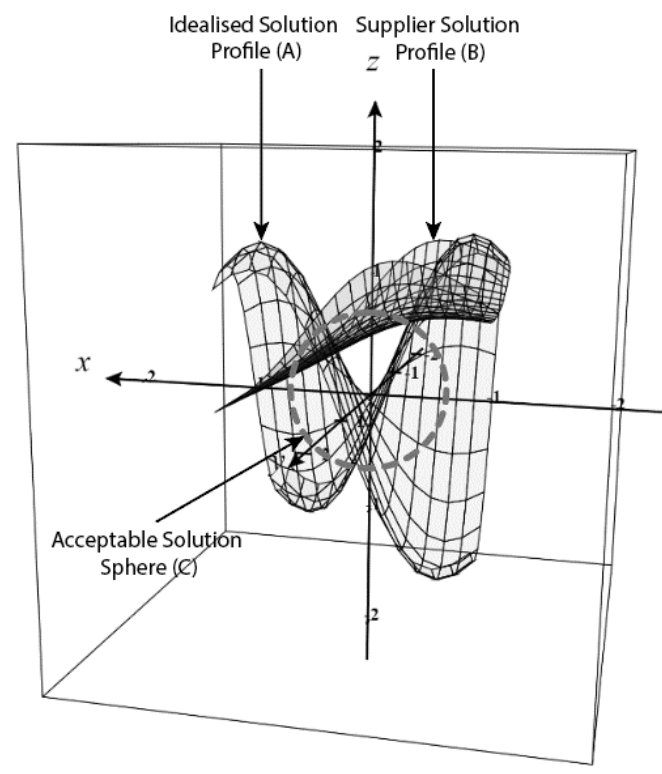

Figure 1-health-climate-economic solution space with "do nothing" solution sphere highlighted.

\section{Explanatory Note}

When evaluating new solutions, "Do Nothing" solutions have already been in operation for a long period of time. This allows the evaluator to "draw" final conclusions about the do nothing climate, cost and health impacts if available. This creates a spherical boundary in the evaluation space corresponding to the performance of the do-nothing against the idealised solution or alternatively, original claims made about the do-nothing solution by the original supplier. This further enhances the body of knowledge of the health service, by providing both the delta between the expected and actual systemic medical device or medicinal performance against the "do nothing" control while facilitates a "real time" equivalent of phase 4 clinical trial for medical devices during the lifetime of the contract.

\section{Introducing the Rapid Viability Test}

For solution candidates, this method creates a rapid viability assessment that filters candidate solution performance and simply needs to compare the Eigenvalues of candidate solutions, including the traditional "do nothing" as part of a 2-phase evaluation to determine if the solution is viable before assessing which candidate solution is most optimal. Specifically, does it have a saddle point? Any solution which does not result in a saddle point can be rejected.

Saddle points can be found using second partial derivative test, which relies on the Eigenvalues of the Hessian matrix to identify the characteristic matrix determinant solved for cubic expressions (10): 


$$
\begin{aligned}
\left|\begin{array}{rrr}
f_{c c c} & f_{c p c} & f_{c p h} \\
f_{p c c} & f_{p p p} & f_{p c h} \\
f_{h c c} & f_{h p c} & f_{h h h}
\end{array}\right| & =f_{c c c}\left(f_{p p p} f_{h h h}-f_{p c h} f_{h p c}\right) \\
& -f_{c p c}\left(f_{p c c} f_{h h h}-f_{p c h} f_{h c c}\right) \\
& +f_{c p h}\left(f_{p c c f_{h p c}}-f_{p p p} f_{h c c}\right)
\end{aligned}
$$

Resulting in no more than 3 Eigenvalues. $E_{1}, E_{2}, E_{3}$.

A saddle is found when $\left\{E \in\left\{E_{1}, E_{2}, E_{3}\right\} \mid \exists E \cdot E>0 \cap\right.$ $\exists E \cdot E<0\}$ and this is the idealised point of maximal value for the procurement. This is similar to assessing cost criteria using a weighted score by assessing supplier offers relative to the cheapest supplier, except the idealised supplier offer becomes the benchmark.

This method of evaluation also naturally lends itself to QALY's defined using the complex plane. Thus, combining methodologies is possible as long as the QALY is correctly applied by the supplier.

It is important to note that this is a necessary but insufficient condition, as this simply knocks out unviable solutions, not evaluates the remaining viability, which must be conducted as a comparison between the remaining candidates.

\section{Conclusion}

Health-climate-economics offers a way to reattach two disparate parts of health service procurement into a complete, circular economic evaluation method that doesn't need to rely on qualitative assessments and includes impacts beyond the visibility of the health-economic models. Rapid viability test allows for a robust shortlisting process and together, these provide a framework for reliable evaluation and inclusion of strategic NHS "net zero" climate goals, and creates a foundation for automated or evidence-assisted evaluation.

\section{Bibliography}

1. Kernick, D P. Introduction to health economics for the medical practitioner. Postgraduate Medical Journal. [Online] 2003. https://pmj.bmj.com/content/79/929/147.

\section{Supply Chain Coordination Limited.}

supplychain.nhs.uk. [Online] https://wwwmedia.supplychain.nhs.uk/media/SCCL_Structu re_Diagram.pdf.

3. Paavola, J. Health impacts of climate change and health and social inequalities in the UK. [Online] 2017. https://ehjournal.biomedcentral.com/articles/10.1186/s12940 -017-0328-z\#MOESM1.

\footnotetext{
4. Jiří Jaromír Klemeš, a,* Yee Van Fan,a and Peng

Jiangb. The energy and environmental footprints of COVID-19 fighting measures - PPE, disinfection, supply chains. [Online] 15 November 2020. https://www.ncbi.nlm.nih.gov/pmc/articles/PMC7450254/.
}

5. UK Government. Parliament Library. Gov.UK. [Online] 2018.

https://assets.publishing.service.gov.uk/government/uploads/ system/uploads/attachment_data/file/218574/ghg-freightguide.pdf.

6. National Health Service. NHS England. england.nhs.uk. [Online] October 2020.

https://www.england.nhs.uk/greenernhs/wpcontent/uploads/sites/51/2020/10/delivering-a-net-zeronational-health-service.pdf.

7. European Heart Journal. Cardiovascular disease burden from ambient air pollution in Europe reassessed using novel hazard ratio functions . [Online] 12 March 2019.

https://academic.oup.com/eurheartj/article/40/20/1590/5372 326.

8. IS Global (Barceolna Institute for Global Health). Barcelona Institute for Global Health. ISGlobal. [Online] 8 August 2019. https://www.isglobal.org/en/-/el-33-de-loscasos-nuevos-de-asma-infantil-en-europa-son-atribuibles-ala-contaminacion-atmosferica.

9. UK Government. UK Government. Gov.uk. [Online] 11 072019.

https://www.gov.uk/government/news/government-targetsparticulate-matter-and-microplastic-pollution-from-cars.

10. Utah Department of Mathmeatics. Utah.edu. [Online] http://www.math.utah.edu/ wortman/1060text-tcf.pdf.

Distribution License: $\underline{\text { CC BY-NC }}$ 